\title{
Cost Bounds and Approximation Ratios of Multicast Light-trees in WDM Networks
}

\author{
Fen Zhou, Miklós Molnár, Bernard Cousin, Member, IEEE, and Chunming Qiao, Fellow, IEEE,
}

\begin{abstract}
The construction of light-trees is one of the principal subproblems for all-optical multicast routing (AOMR) in sparse splitting Wavelength Division Multiplexing (WDM) networks. Due to the light splitting constraint and the absence of wavelength converters, several light-trees may be required to establish a multicast session. However, the computation of the cost-optimal multicast light-trees is NP. hard. In this paper, first we study the cost bounds of the lighttrees built for a multicast session in unweighted WDM networks. Then, partially based on this result, the approximation ratios of some classical multicast light-tree computation algorithms, i.e., Reroute-to-Source (R2S) and Member-Only (MO) algorithms are derived in both unweighted and nonequally weighted WDM networks. Moreover, integer linear programming (ILP) formulations are introduced and carried out to search the optimal light-trees for multicast routing. The cost bounds and approximation ratios of $R 2 S$ and MO algorithms in some candidate WDM backbone networks are examined through simulations.
\end{abstract}

Index Terms-Cost Bound; Approximation Ratio; Lighttree, All-Optical Multicast Routing (AOMR); WDM Network; Sparse Splitting.

\section{INTRODUCTION}

A LL-optical multicast routing (AOMR) [1] is to determine a set of lightpaths from a source to the multicast members of the same session in a WDM network. The light-tree concept is introduced in [2] to minimize the number of wavelength channels and transceivers for alloptical multicasting. Branching nodes in a light-tree should be equipped with light splitters to support multicasting. However, in sparse splitting [3] WDM networks, there are two kinds of nodes: Multicast-Capable nodes (MC [3], i.e. the nodes equipped with light splitters) and Multicast-I ncapable nodes (MI [3], i.e. the nodes without light splitters). An MC node is able to replicate the data packets in the optical domain via light splitting and send the split light beam to all the outgoing ports. While an MI node cannot split but generally has the Tap-and-Continue ( $\mathrm{TaC}$ [4]) capability. The TaC permits to tap a small amount of optical power from the incoming light beam for local usage and forward the rest to only one outgoing port. Although one tree is sufficient to span all the multicast destinations in a network without splitting constraints, minimizing the cost of the multicast tree is already a Steiner-Problem which is NPcomplete [7]. Due to sparse splitting, lack of wavelength

Part of this work has been presented in [5] on IFIP Networking 2010 conference and in [6] on IEEE GLOBECOM 2010 conference.

Fen Zhou and Bernard Cousin are with the IRISA lab (a joint research center of INSA Rennes, University of Rennes 1 and CNRS), campus de beaulieu, Rennes 35042, France (e-mail: \{fen.zhou, bcousin\}@irisa.fr).

Miklós Molnár is with the LIRMM Iab, 161 rue Ada, University of Montpellier II, Montpellier 34392, France (e-mail: miklos.molnar@irmm.fr).

Chunming Qiao is with the department of computer science \& engineering in the University at Buffalo, NY 14260-2000, USA (email: qiao@computer.org). converters, as well as continuous wavelength and distinct wavelength constraints [8], one light-tree may not be able to cover all the members of a multicast session while several ones may be required, i.e., a light-forest [9]. As a result, it is even harder to optimize the total wavelength channel cost for a multicast session.

Although many light-tree computation heuristics have been proposed recently [7], [9]-[12], none of them has addressed the cost bound of multicast light-trees in sparse splitting WDM networks, let alone the approximation ratio ${ }^{11}$ of the heuristic algorithms. Since the wavelength channel cost is a very important metric for the selection of the multicast light-trees, it is very critical to know at least the cost bound of the light-trees, which could be referenced when designing a WDM network. In [7], a heuristic is proposed to construct multicast light-trees with QoS guarantee and the cost upper bound of the light-trees is given. However, in [7] it is supposed that all the network nodes are equipped with costly light splitters, while it is not realistic in large WDM mesh networks due to the high cost and complex architecture of light splitters. Literature [13] also gives a cost upper bound of $\frac{N^{2}}{4}$ for the multicast light-trees, where $N$ denotes the number of nodes in the network. However, the cost bound in [13] has the following two shortcomings. First it is derived on the hypothesis that the set of multicast light-trees computed for a multicast session still retain a tree structure in the IP layer (i.e., when all these lighttrees are merged together). In fact, this hypothesis is not always held as demonstrated in the following example. A multicast session with source $s$ and destinations $d_{1}, d_{2}$ and $d_{3}$ is required in a sparse splitting optical network shown in Fig. 11 with solid line. Since node $d_{3}$ is an MI node, two light-trees (i.e., $L T_{1}$ (dotted line) and $L T_{2}$ (dashed line)) on two different wavelengths may be computed. As we can see the IP layer of the merged $L T_{1}$ and $L T_{2}$ are drawn in Fig. 1 with solid line, which is the same as the network topology. Obviously, it is not a tree but a cycle. Second, the bound $\frac{N^{2}}{4}$ in [13] seems to be too large for small size multicast sessions, e.g., a multicast session with a source and only two destinations.

For the above reasons, the first contribution of this paper is to give a more accurate bound for wavelength channel cost of multicast light-trees, which is based on our previous result in [5]. It is valid for most of the multicast routing algorithms under sparse splitting constraint, even if the IP layer of the set of multicast light-trees does not retain the tree structure (e.g, the iterative multicast routing algorithms as MemberOnly [9]). Costly and complex wavelength converters are supposed to be unavailable, and an equal cost of 1 unit hop-

${ }^{1} \mathrm{~A}$ heuristic algorithm has an approximation ratio of $\rho$ in network $G$, if it can be guaranteed that for all possible multicast sessions in $G$ the total cost of the multicast light-forest computed by the heuristic algorithm is at most $\rho$ times worse than the total cost of the optimal solution. 
TABLE I

Summary of the Approximation Patios obtained in Sparse Splitting WDM Networks

\begin{tabular}{|c|c|c|c|c|}
\hline Algorithm & Unweighted WDM network & \multicolumn{3}{|c|}{ Non-equally weighted WDM network } \\
\hline & $1 \leq K \leq N$ & $1 \leq K<\frac{\sqrt{16 N+49}-7}{2}$ & $\frac{\sqrt{16 N+49}-7}{2} \leq K<\frac{N}{2}$ & $\frac{N}{2} \leq K<N$ \\
\hline MO & $\rho(M O) \leq\left(K^{2}+3 K\right) / 4$ & $\rho(M O) \leq\left(K^{2}+3 K\right) / 4$ & $\rho(M O) \leq N-K$ & $\rho(M O) \leq \frac{\left\lfloor\frac{N}{4}\right\rfloor}{K}$ \\
\hline $\mathrm{R} 2 \mathrm{~S}$ & $\rho(R 2 S)=K$ & \multicolumn{2}{|c|}{$\rho(R 2 S) \leq K$} & $\rho(R 2 S) \leq \frac{\left\lfloor\frac{N-}{4}\right\rfloor}{K}$ \\
\hline
\end{tabular}

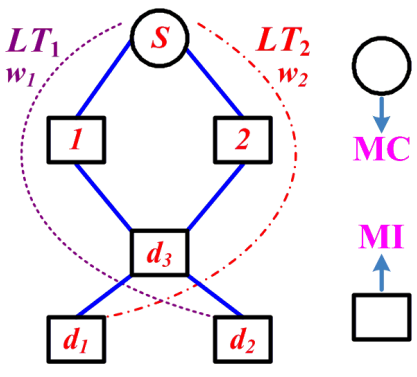

Fig. 1. An example sparse splitting WDM network

count-cost is assumed over all the fiber links in the network. We prove that the total cost of a multicast session is upper bounded to (1) $K(N-K)$, when $K<\frac{N}{2}$; (2) $\left\lfloor\frac{N^{2}}{4}\right\rfloor$, when $K \geq \frac{N}{2}$, where $K$ is the number of destinations in the multicast session and $N$ is the number of nodes in the network. Besides, the wavelength channel cost is lower limited to $K$. Moreover, in unweighted WDM rings the optimal multicast light-tree has a total cost inferior to $N-\left\lceil\frac{N}{K+1}\right\rceil$.

Solving the Steiner problem, the Shortest Path Tree algorithm approximates the optimal solution with a ratio of $K$, which is the number of destinations to be covered. A better heuristic algorithm named Minimum Path Heuristic [14] guarantees the result cost with a ratio of $2\left(1-\frac{1}{K+1}\right)$ [15]. Solving the multicast routing problem in sparse splitting WDM networks, the Reroute-to-Source (R2S) and MemberOnly (MO) al gorithms are proposed in [9]. These two heuristics are the variant algorithms of the Shortest Path Tree and Minimum Path Heuristic in WDM networks. Will they retain the same approximation ratios as for solving the Steiner problem? Thus, the second contribution of this paper is to investigate their approximation ratios in both unweighted and non-equally weighted WDM networks, which is summarized in Table [] While our previous work [6] only considered the latter case.

Moreover, cost bounds and approximation ratios of multicast light-trees in some candidate all-optical backbone networks are examined through simulations. Integer Linear Programming (ILP) formulations are proposed to find the optimal multicast light-trees. MO and R2S [9] algorithms are also implemented in the simulation.

The rest of this paper is organized as follows. System model is given and the multicast routing problem is formulated in Section W Then the cost bound of multicast lighttrees in WDM mesh network is discussed in Section III] After that, the cost bound of multicast light-trees in WDM rings is investigated in Section [V] Furthermore, the approximation ratios of two dassical multicast routing al gorithms are derived in Section $\nabla$ To search the optimal solution for sparse splitting multicast routing, the ILP formulations are introduced in Section VI. The proposed cost bounds and ap- proximation ratios are evaluated in Section VII by extensive simulations. Finally, we conclude the paper in Section VIII. It should be noticed that most proofs of theorems and lemmas are included in the appendixes in order to have a good flow in the paper.

\section{Multicast Routing With Sparse Splitting}

\section{A. Multicast Routing Problem}

Multicast routing involves a source and a set of destinations. In sparse splitting WDM networks, a set of light-trees is employed to distribute messages from the source to all the group members simultaneously. The objective of studying multicast routing in WDM networks is to minimize the wavelength channel cost while fulfilling a multicast session. The computation of light-trees for a multicast session generally has the following principles.

1) Due to sparse splitting and absence of wavelength conversion, in a light-tree, the degree of an MI node cannot exceed two. In consequence some destinations cannot be included in the same light-tree. Thus, several light-trees on different wavelengths may be required for one multicast session.

2) Among the light-trees built for a multicast session, one destination may be spanned (used to forward the incoming light beam to other destination nodes) by several light-trees, but it should be served (used to receive messages from the source) by only one lighttree. (e.g., $d_{3}$ in Fig. [1] is spanned by both $L T_{1}$ and $L T_{2}$ to forward the incoming light beam to $d_{2}$ and $d_{1}$ respectively. Thus, it must tap the light beam only once for recovering multicast messages either in $L T_{1}$ or in $L T_{2}$ ).

3) Since the number of wavelengths supported per fiber link is limited, the maximum number of wavelengths required and the traffic congestion in a fiber link should be taken into account during the selection of multicast light-trees. Thus, if a set of destinations $D$ have been spanned by a light-tree $L T_{1}, D \subseteq L T_{1}$, it is entirely useless to construct another light-tree $L T_{2}$ to serve and only serve the destinations in subset $D_{i}$, with $D_{i} \subseteq D$. This is because that destinations in $D_{i}$ could be served directly in $L T_{1}$. For instance, three light-trees $L T_{1}, L T_{2}$ and $L T_{3}$ are computed to serve $d_{1}, d_{2}, d_{3}$ respectively, where $L T_{1}$ only contains $d_{1}, d_{2}, L T_{2}$ only contains $d_{2}, d_{3}$ and $L T_{3}$ only contains $d_{3}, d_{1}$. However, $L T_{3}$, for instance, should be eliminated since $d_{3}$ is spanned in $L T_{2}$ and can be served directly in $L T_{2}$ instead of using the tree $L T_{3}$.

\section{B. System Model}

A sparse splitting WDM network can be modeled by an undirected graph $G(V, E, c) . V$ represents the vertex-set of 
$G,|V|=N$. Each node $v \in V$ is either an $\mathrm{MI}$ or an MC node. $E$ represents the edge-set of $G$, which corresponds to the fiber links between the nodes in the network. Each edge $e \in E$ is consisted of two optical fibers for opposite direction communications. And $e$ is associated with a cost function $c(e)$. Function $c$ is additive over the links of a lightpath $L P(u, v)$ between two nodes $u$ and $v$, i.e.,

$$
c(L P(u, v))=\sum_{e \in L P(u, v)} c(e)
$$

We consider a multicast session $m s(s, D)$, which requests for setting up a light distribution structure (i.e., light-forest) under optical constraint (i.e., wavelength continuity, distinct wavelength, sparse splitting and lack of wavelength conversion constraints) from the source $s$ to a group of destinations $D$. Let $K$ be the number of destinations, $K=|D|$. Without loss of generality, it is assumed that $k$ light-trees $L T_{i}\left(s, D_{i}\right)$ are required to span all the destinations involved in a multicast session $m s(s, D)$, where $i \in[1, k]$. It holds true that

$$
1 \leq k \leq K \leq N-1
$$

Although the $i^{\text {th }}$ light-tree $L T_{i}\left(s, D_{i}\right)$ may span some destinations already spanned in the previous light-trees, $D_{i}$ is used to denote exclusively the set of newly served destinations in $L T_{i}\left(s, D_{i}\right)$. Since all the destinations in $D$ are served by $k$ light-trees and each destination should be served only once, we obtain

$$
D=\bigcup_{i=1}^{k} D_{i}
$$

These $k$ sets of destinations $D_{i}$ are disjoint, i.e.,

$$
\forall i, j \in[1, k] \text { and } i \neq j, D_{i} \cap D_{j}=\varnothing
$$

Let a positive integer $K_{i}=\left|D_{i}\right|$ denote the size of the subset $D_{i}$, then we have

$$
\sum_{i=1}^{k} K_{i}=|D|=K
$$

The total cost $\mathcal{C}$ of a multicast session $m s(s, D)$ is defined as the wavelength channel cost of the light-trees built to serve all the destinations in set $D$. It can be calculated by

$$
\begin{aligned}
\mathcal{C}(m s(s, D)) & =\sum_{i=1}^{k} c\left[L T_{i}\left(s, D_{i}\right)\right] \\
& =\sum_{i=1}^{k} \sum_{e \in L T_{i}\left(s, D_{i}\right)} c(e)
\end{aligned}
$$

\section{COSt Bounds of Multicast Light-TREES IN WDM MESH NETWORKS}

In this section, we will study the cost bounds of light-trees in unweighted WDM networks with two different light splitting configurations: full light splitting and sparse splitting. Let $S R=N_{M C} / N$ be the ratio of MC nodes in the network. For the full light splitting case $S R=1$, and for the sparse splitting case $0 \leq S R<1$. For simplicity, it is assumed that all links have the following cost function in unweighted
WDM networks:

$$
c(e)=1 \text { unit hop-count-cost } 2
$$

Thus,

$$
\mathcal{C}(m s(s, D))=\sum_{i=1}^{k} \sum_{e \in L T_{i}\left(s, D_{i}\right)} 1
$$

\section{A. Full Light Splitting WDM Networks}

In the case that all network nodes are equipped with light splitters, each node could act as a branching node in a light-tree. Hence, one light-tree is sufficient to span all the multicast members. It is a Steiner-problem which tries to find a minimum partial spanning tree covering the source and all the multicast members. In a light-tree, there are at most $N$ nodes when all the network nodes are spanned (i.e., when $\{v \mid v \in L T\}=V$ ), and at least $K+1$ nodes if and only if the light-tree just contains the source and the multicast members (i.e. when $\{v \mid v \in L T\}=\{s\} \cup D$ ). So, the cost of the multicast light-tree is bounded to

$$
K \leq \mathcal{C}(m s(s, D)) \leq N-1
$$

To minimize the total cost in full light splitting case, the Minimum Path heuristic [14] and the Distance Network heuristic [16] can be good choices, since they are guaranteed to get a light-tree with a total wavelength channel cost no more than $2\left(1-\frac{1}{K+1}\right)$ times that of the optimal Steiner tree [15], [16]. i.e.

$$
\mathcal{C}(m s(s, D)) \leq 2\left(1-\frac{1}{K+1}\right) \times C_{O p t}
$$

where $C_{O p t}$ denotes the wavelength channel cost of the Steiner tree for $m s(s, D)$.

\section{B. Sparse Splitting WDM Networks}

In the case of sparse splitting, only a subset of nodes can act as branching nodes in a light-trees. One light-tree may not be sufficient to accommodate all the group members simultaneously. Generally, several light-trees should be employed.

Lemma 1: $\forall j \in[1, k]$, the cost of the $j^{\text {th }}$ light-tree holds

$$
K_{j}=\left|D_{j}\right| \leq c\left(L T_{j}\left(s, D_{j}\right)\right) \leq N-k
$$

Theorem 1: In sparse splitting WDM networks, the total cost of the light-trees built for the multicast session $m s(s, D)$ satisfies

$$
K \leq \mathcal{C}(m s(s, D)) \leq \begin{cases}K(N-K), & K<\frac{N}{2} \\ \left\lfloor\frac{N^{2}}{4}\right\rfloor, & K \geq \frac{N}{2}\end{cases}
$$

\section{COST BOUND OF MULTICAST LIGHT-TREES IN UNWEIGHTED WDM RINGS}

\section{A. Multicast Light-tree in unweighted WDM Rings}

In WDM rings, all the nodes are mandatorily equipped with $\mathrm{TaC}$ [4] capability, one light-tree is able to span all the multicast members. The multicast light-tree in a WDM ring consists of either a lightpath or two edge disjoint lightpaths originating from the same source. In an $N$-node WDM ring, the cost of the multicast light-tree for multicast session $m s(s, D)$ is subject to

$$
K \leq \mathcal{C}(m s(s, D)) \leq N-1
$$

${ }^{2}$ For instance, in an unweighted WDM network, each link is an optical fiber of $10 \mathrm{~km} .1$ unit hop-count-cost can be considered as the wavelength channel cost in a $10 \mathrm{~km}$ optical fiber. 
B. Optimal Multicast Light-tree in unweighted WDM Rings

Different from WDM mesh networks, minimizing the cost of the multicast light-tree in a WDM ring is very simple. The minimum spanning tree for the multicast members is the optimal solution. Here, we use the concept gap introduced in [17], [18]. A gap is a path between two adjacent multicast members in $\{s\} \cup D$ so that no other members are involved in this path. The optimal multicast light-tree can be obtained by removing the biggest gap from the ring [17].

Theorem 2: In a WDM ring, the cost of the optimal lighttree for multicast session $m s(s, D)$ complies

$$
K \leq \mathcal{C}(m s(s, D)) \leq N-\left\lceil\frac{N}{K+1}\right\rceil
$$

\section{Approximation Ratios of the heuristic ALGORITHMS FOR SPARSE SPLITTING MULTICAST ROUTING}

Like the Steiner problem, it is NP-hard to find the lighttrees with the optimal cost for multicast routing in sparse splitting WDM networks. This is why many heuristic algorithms have been proposed to solve this problem in polynomial time. In order to guarantee the quality of the resultant light-trees, it is imperative to determine the cost approximation ratios of the proposed heuristic solutions. The approximation ratio $\rho(H)$ of a heuristic algorithm $H$ in WDM network $G$ can be defined as follows: for any possible multicast session $m s(s, D)$ in $G$, let $\mathcal{C}(H)$ be the total cost of the multicast light-forest computed by $H$ and let $C_{O p t}$ be the total cost of the optimal solution (the solution with the minimized cost), $\rho(H)$ is the tight upper bound of the equation below

$$
1 \leq \frac{\mathcal{C}(H)}{C_{O p t}} \leq \rho(H), \forall m s(s, D) \text { in } G
$$

Nevertheless, the approximation ratios of heuristic algorithms have not been investigated before. In this section, we try to deduce the approximation ratios of two dassical light-trees computation heuristics namely Reroute-to-Source (R2S) and Member-Only (MO) [9]. Define $C_{O p t}$ as the optimal cost of the light-trees fulfilling the multicast session $m s(s, D)$, and let $\rho(\cdot)$ denote the cost approximation ratio of a heuristic solution. Specially, we discuss the approximation ratios of these algorithms in two types of WDM networks: unweighted one and non-equally weighted one. In the first case, all the link costs are set to be 1 unit hop-count-cost as shown in Eq. (7). While in the latter case, the link cost can be an arbitrary positive number.

At first, we give the general approximation ratios in unweitghted WDM mesh networks and some special topologies.

Lemma 2: Given that WDM network $G$ is unweighted, if an all-optical multicast routing al gorithm $A O M R$ follows the assumptions in Subsection $11-A$ then its approximation ratio holds

$$
\rho(A O M R) \leq\left\{\begin{array}{cl}
N-K & 1 \leq K<\frac{N}{2} \\
\frac{\left\lfloor\frac{N^{2}}{4}\right\rfloor}{K} & \frac{N}{2} \leq K \leq N
\end{array}\right.
$$

Proof: In unweighted WDM networks, Theorem 1 gives both the lower bound and the upper bound for the lightforest of one multicast session. Obviously, the optimal cost of a light-forest should be no less than the lower bound $K$, while the cost of any light-forest can not be beyond the upper bound. Hence, the approximation ratio of the algorithm can not be greater than the value of the upper bound divided by the lower bound.

This theorem is valid for both Reroute-to-Source and Member-Only al gorithms, since they respect the sparse splitting constraint and follow the aforementioned assumptions. It should also be noted that both Reroute-to-Source and Member-Only algorithms are capable of finding the costoptimal light-forest in some special topologies, for instance a tree, a simple path, and etc.

Lemma 3: Given the WDM network $G$ in which there is one and only one path between each pair of nodes, the approximation ratios of Reroute-to-Source and Member-Only algorithms are equal to 1.

Proof: As there is only one path between each pair of nodes, any solution will find the identical light-forest to realize a multicast session.

\section{A. Rerouteto-Source Algorithm}

Reroute-to-Source algorithm constructs the shortest path tree rooted at the source, then it checks the splitting capacity of the branching nodes. If a branching node is an MI node, the algorithm cuts all but one downstream branch. The affected leaf destinations rejoin the light-tree along a shortest path to the source on another wavelength.

Theorem 3: Given that WDM network $G$ is non-equallyweighted, the Reroute-to-Source algorithm [9] provides an approximation ratio of $\rho(R 2 S)=K$ for multicast routing with sparse splitting constraint.

Theorem 4: Given that WDM network $G$ is unweighted,

$$
\rho(R 2 S) \leq\left\{\begin{array}{cc}
K & 1 \leq K<\frac{N}{2} \\
\frac{\left\lfloor\frac{N^{2}}{4}\right\rfloor}{K} & \frac{N}{2} \leq K \leq N-1
\end{array}\right.
$$

\section{B. Member-Only Algorithm}

According to Member-Only (MO) algorithm [9], the shortest path between each pair of nodes is precalculated and stored in a table. Then, the computation of the light-trees for a multicast request is done iteratively as shown in Algorithm [1]

$M C \_S E T$ : includes source node, MC nodes and the leaf MI nodes. They may be used to span the light-tree $L T$ and, thus are also called connector nodes in $L T$.

$M I$ SET: includes only the non-leaf MI nodes, whose splitting capability is exhausted. Hence, these nodes are not able to connect a new destination to the subtree $L T$.

$D$ : includes unserved multicast members which are neither joined to the current light-tree $L T$ nor to the previously constructed multicast light-trees.

At each step $i+1$, try to find the shortest paths between the destinations $d \in D$ and the connector nodes $c \in M C \_S E T$ of light-tree $L T_{i}$, such that they do not involve any $\mathrm{TaC}$ capability exhausted nodes in MI_SET. Among them, the constraint-satisfying shortest path $S P(d, c)$ with the smallest cost is selected. Then generate $L T_{i+1}$ by adding $S P(d, c)$ to $L T_{i}$. In case that no such destination can be found, begin a new light-tree rooted at the source. Member-Only algorithm is an adjustment of the famous Minimum Path Heuristic (MPH) proposed for the Steiner problem. As mentioned in Section IIII MPH is able to approximate the Steiner tree with a ratio smaller than 2 . However, by adjusting MPH for 


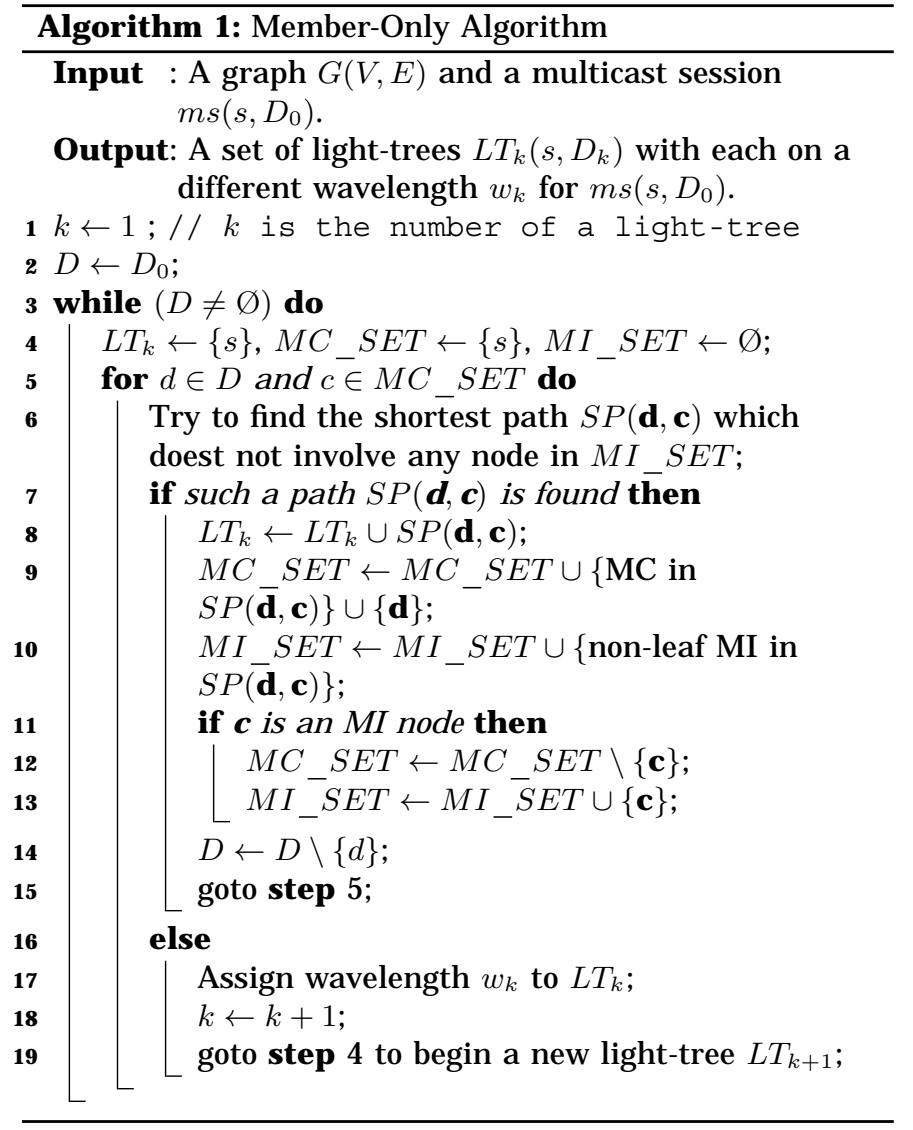

multicast routing under sparse splitting constraint, MemberOnly algorithm results in different approximation ratios.

Theorem 5: Given any kind of WDM networks $G$, the Member-Only algorithm provides an approximation ratio $\rho(M O) \leq \frac{K^{2}+3 K}{4}$ for sparse splitting multicast routing.

Theorem 6: Given that WDM network $G$ is unweighted, then

$$
\rho(M O) \leq\left\{\begin{array}{cc}
\frac{1}{4}\left(K^{2}+3 K\right) & 1 \leq K<\frac{\sqrt{16 N+49}-7}{2} \\
N-K & \frac{\sqrt{16 N+49}-7}{2} \leq K<\frac{N}{2} \\
\frac{\left\lfloor\frac{N^{2}}{4}\right\rfloor}{K} & \frac{N}{2} \leq K \leq N-1
\end{array}\right.
$$

\section{ILP FORMULATION}

Since minimizing the total cost of the light-forest for a multicast session is NP-hard, the integer linear programming (ILP) method is applied to search the optimal solution.

\section{Notations and Variables}

$W \quad$ : The wavelengths supported per fiber.

$\lambda \quad$ : A wavelength $\lambda \in W$.

In $(m) \quad:$ The set of nodes leading an edge to node $m$.

$\operatorname{Out}(m) \quad:$ The set of nodes to which $m$ is connected.

$\operatorname{Deg}(m) \quad$ : The degree of node $m$.

$\operatorname{link}(m, n) \quad$ : The directed link from node $m$ to node $n$.

$c_{m, n} \quad:$ The cost of $\operatorname{link}(m, n)$.

$L_{m, n}(\lambda) \quad$ : Equals to 1 if multicast request $m s(s, D)$ uses wavelength $\lambda$ on $\operatorname{link}(m, n)$, equals to 0 otherwise.

$U_{m, n}^{d}(\lambda) \quad$ : Equals to 1 if $\operatorname{link}(m, n)$ is used on wavelength $\lambda$ in the lightpath from $d$ to the source $s$, equals to 0 , otherwise.
The objective of the studied sparse splitting multicast routing problem is to minimize the wavelength channel cost of the light-trees built for a multicast session $m s(s, D)$. It can be formulated as follows:

$$
\text { Minimize : } \sum_{\lambda \in W} \sum_{m \in V} \sum_{n \in \operatorname{In}(m)} L_{n, m}(\lambda) \cdot c_{n, m}
$$

The objective function is subject to a set of constraints, which are listed below:

\section{A. Multicast Light-tree Constraints}

Source Constraints:

$$
\begin{array}{r}
\sum_{\lambda \in W} \sum_{n \in \operatorname{In}(s)} L_{n, s}(\lambda)=0 \\
1 \leq \sum_{\lambda \in W} \sum_{n \in \text { Out }(s)} L_{s, n}(\lambda) \leq|D|
\end{array}
$$

Constraints (20) and (21) ensure that the light-trees for multicast session $m s(s, D)$ are rooted at the source node $s$. In a light-tree, $s$ must not have any input link, but should have at least one output link. And the number of outgoing links from $s$ should not go beyond the number of sink nodes, i.e., $|D|$.

Destinations Constraints:

$$
1 \leq \sum_{\lambda \in W} \sum_{n \in \operatorname{In}(d)} L_{n, d}(\lambda) \leq|D|, \quad \forall d \in D
$$

Constraint (22) guarantees that each destination node sinks at least one incoming light beam. Since some destinations, which act an intermediate node in a light-tree, will forward the incoming light beam to successor destinations, a destination node $d$ can receive at most $|D|$ light beams on all the wavelength layers. However, this constraint cannot ensure that destination $d$ is reachable from the source $s$, which will be illustrated later.

Input Constraint:

$$
\sum_{n \in I n(m)} L_{n, m}(\lambda) \leq 1, \quad \forall \lambda \in W, \text { and } \forall m \in V
$$

Equation (23) indicates that each node (except the source $s$ ) in a light-tree has and only has one predecessor. Nevertheless, this constraint can not guarantee that the resultant structure is a set of light-trees, due to the fact that loops can not be avoided (refer to Fig. 2).

Leaf Nodes Constraint:

$$
\begin{array}{r}
\sum_{n \in O u t(m)} L_{m, n}(\lambda) \geq \sum_{n \in I n(m)} L_{n, m}(\lambda) \\
\forall \lambda \in W, \forall m \in V \text { and } m \notin D
\end{array}
$$

Constraint (24) ensures that only the destination nodes can be leaf nodes in a light-tree while the non-member nodes can not.

Sparse Splitting Constraints:

$$
\begin{array}{r}
\sum_{n \in \text { Out }(m)} L_{m, n}(\lambda) \leq R \times \sum_{n \in I n(m)} L_{n, m}(\lambda) \\
\forall \lambda \in W, \forall m \in V \text { and } m \neq s
\end{array}
$$

where

$$
\begin{cases}R=1, & \text { if } m \text { is an } \mathrm{MI} \text { node } \\ R=\operatorname{Deg}(m)-1, & \text { if } m \text { is an } \mathrm{MC} \text { node }\end{cases}
$$




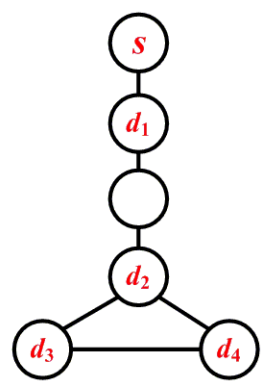

(a)

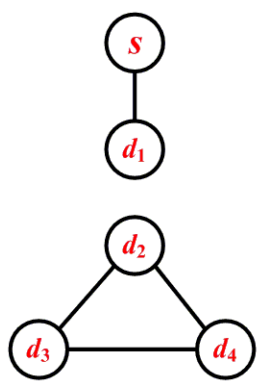

(b)
Fig. 2. A contradict example with a loop in the result light-tree: (a) The network topology; (b) The result

Constraint (25) together with constraint (24) indicates the splitting capabilities of the nodes. If a node $m$ is spanned in a light-tree, then the number of outgoing links from $m$ is equal to 1 for an $\mathrm{MI}$ node and less than $\operatorname{Deg}(m)-1$ for an MC node. Otherwise, it must be 0 .

Only with the light-tree structure constraints developed above [19], [20], one can not guarantee that each light-tree of the resultant light-forest should be connected and loop free. An contradictory example is given next. Suppose we just employ the light-tree constraints formulation to find the light-trees for a multicast session $m s\left(s,\left(d_{1}-d_{4}\right)\right)$ in topology Fig. 2(a). The result in Fig. 2(b) uses some wavelength $\lambda_{1}$, where $L_{s, d_{1}}\left(\lambda_{1}\right)=1, L_{d_{2}, d_{3}}\left(\lambda_{1}\right)=1, L_{d_{3}, d_{4}}\left(\lambda_{1}\right)=1$, $L_{d_{4}, d_{2}}\left(\lambda_{1}\right)=1$ and all the other variables $L_{m, n}(\lambda)$ are zero. It is true that all the constraints from (20) to [25) are satisfied in this result. Besides, the wavelength channel cost of the result is optimal. Unfortunately, this result has a loop $d_{2}-d_{3}-d_{4}-d_{2}$ and three destinations are separated from the source node $s$. Thereby, the proposed light-trees constraints are not sufficient to guarantee the resultant lighttree structure. This is why next the destinations reachability constraints are introduced to solve these problems.

\section{B. Destination Nodes Reachability Constraints}

Source node:

$$
\begin{gathered}
\sum_{n \in \operatorname{In}(s)} U_{n, s}^{d}(\lambda)=0, \quad \forall \lambda \in W, \text { and } \forall d \in D \\
1 \leq \sum_{\lambda \in W} \sum_{n \in \text { Out }(s)} U_{s, n}^{d}(\lambda) \leq|D|, \quad \forall d \in D
\end{gathered}
$$

Similar to constraint (20), Eq. (27) gives the constraint that no link leading to the source will be employed to serve destinations in the light-trees.

Equation (28) ensures that all the destination nodes could be reached from the source node $s$ in the light-trees. By combining Eqs. (23) and (28), the loops can be avoided. Still refer to the contradictory example aforementioned, the result in FigL(b) does not satisfy constraint [28), since destination nodes $d_{2}-d_{4}$ can not be reached from the source node $s$.

Destination nodes autocorrelation:

$$
\begin{gathered}
\sum_{n \in O u t(d)} U_{d, n}^{d}(\lambda)=0, \quad \forall \lambda \in W, \text { and } \forall d \in D \\
\sum_{n \in \operatorname{In}(d)} U_{n, d}^{d}(\lambda) \leq 1, \quad \forall \lambda \in W, \text { and } \forall d \in D
\end{gathered}
$$

$$
1 \leq \sum_{\lambda \in W} \sum_{n \in \operatorname{In}(d)} U_{n, d}^{d}(\lambda) \leq|D|-1, \quad \forall d \in D
$$

Constraint (29) avoids the loops of destinations, such as that in Fig[(b). Constraints (30) and (31) make sure that each destination has one and only one input link in a light-tree, which are equivalent to constraints (23) and (22) respectively.

Non-member nodes and destination nodes cross correlation:

$$
\begin{array}{r}
\sum_{n \in O u t(m)} U_{m, n}^{d}(\lambda)=\sum_{n \in \operatorname{In}(m)} U_{n, m}^{d}(\lambda) \leq 1 \\
\forall \lambda \in W, \forall d \in D, \forall m \in V \text { and } m \neq s, d \\
\sum_{\lambda \in W} \sum_{n \in O u t(m)} U_{m, n}^{d}(\lambda) \leq|D| \\
\forall \lambda \in W, \forall d \in D, \forall m \in V \text { and } m \neq s, d
\end{array}
$$

The distinct wavelength constraint is illustrated by Eq. 32). It ensures that one link can be used at most once on one wavelength, and will be used at most $|D|$ times to establish multicast session $m s(s, D)$ on all the wavelengths which is expressed by Eq. (33).

\section{Relationship between $L_{m, n}(\lambda)$ and $U_{m, n}^{d}(\lambda)$}

In order to avoid loops in the resultant light-trees, variable $U_{m, n}^{d}(\lambda)$ is employed to restrict variable $L_{m, n}(\lambda)$. Their relations are shown in Eqs. (34) and (35).

$$
\begin{gathered}
L_{m, n}(\lambda) \leq \sum_{d \in D} U_{m, n}^{d}(\lambda), \forall \lambda \in W, \text { and } \forall m, n \in V \\
U_{m, n}^{d}(\lambda) \leq L_{m, n}(\lambda), \forall \lambda \in W, \forall m, n \in V \text {, and } \forall d \in D \\
\text { VII. SIMULATION AND NUMERICAL RESULTS }
\end{gathered}
$$

In this section, simulations are conducted to compute the multicast light-trees in sparse splitting WDM mesh networks. ILP formulations are implemented by Cplex [21], while Member-Only and Reroute-to-Source are conducted in $\mathrm{C}++$ with LEDA package [22]. Since the proposed cost bounds and the approximation ratios of Member-Only and Reroute-to-Source algorithms only correspond to the worst or extreme cases, they may only appear in special topologies with special configurations. Hence, here we do not mean to verify the accuracy of the proposed bounds and approximation ratios. Instead, the numerical results are used to show the quality of the resultant light-trees when applying the Member-Only and the Reroute-to-Source algorithms in some popular candidate WDM backbone networks like 14 nodes NSF network [10] and 28 nodes USA Longhaul network [10].

\section{A. Cost Bounds of Multicast Light-trees}

Member-Only (MO) and Reroute-to-Source (R2S) algorithms are conducted in unweighted NSF network (maximal nodal degree is 4) and unweighted USA Longhaul network (maximal nodal degree is 5). All the links are associated with an identical cost of 1 hop-count-cost. Since the worst case of the cost bound occurs when there is no light splitters in the network, we configure the network without light splitters. The source and multicast members are assumed to be distributed uniformly over the topology. The cost bounds of the multicast light-trees computed by $\mathrm{MO}$ and R2S heuristics 


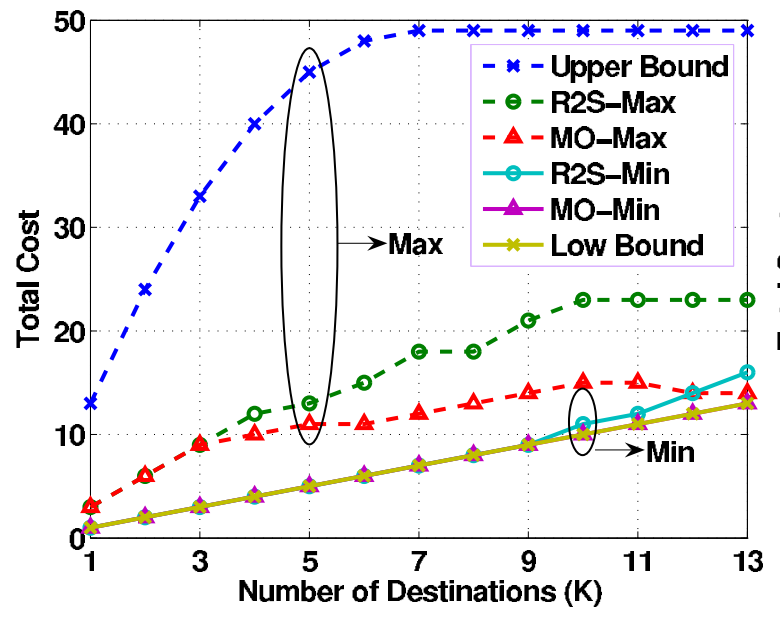

(a) NSF Network

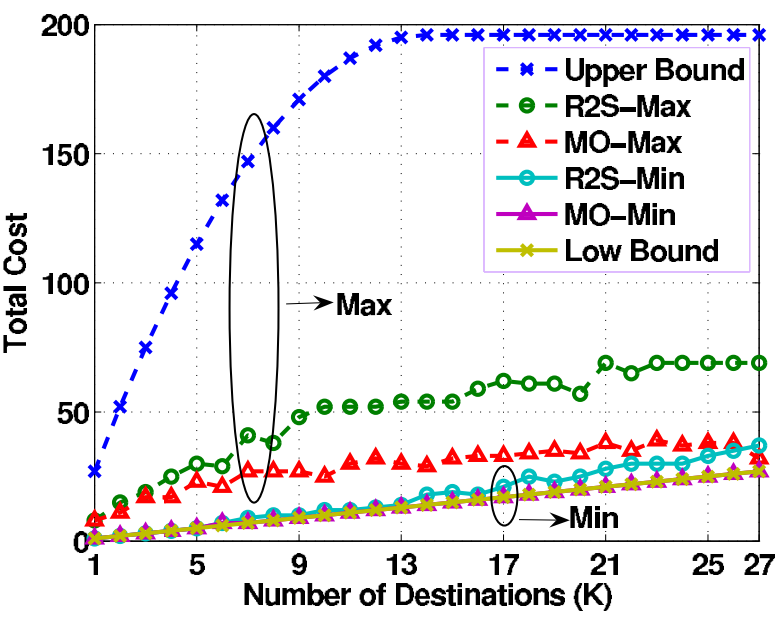

(b) Longhaul Network

Fig. 3. The Cost Bound of multicast light-trees when the number of destinations $K$ varies

TABLE II

COMPARISON OF COST BOUNDS IN NSF NETWORK

\begin{tabular}{|c||c|c|c|c|c|}
\hline$|D|=K$ & LB & ILP & MO & R2S & UB \\
\hline 2 & 2 & 3.2 & 3.2 & 3.6 & 24 \\
\hline 3 & 3 & 4.5 & 4.6 & 5.2 & 33 \\
\hline 4 & 4 & 5.7 & 5.7 & 6.7 & 40 \\
\hline 5 & 5 & 6.7 & 6.9 & 8.2 & 45 \\
\hline 6 & 6 & 8.2 & 8.5 & 9.1 & 48 \\
\hline 7 & 7 & 8.3 & 8.5 & 10.9 & 49 \\
\hline 8 & 8 & 8.7 & 9.3 & 11.7 & 49 \\
\hline 9 & 9 & 9.6 & 10.1 & 12.3 & 49 \\
\hline 10 & 10 & 10.8 & 11.1 & 15 & 49 \\
\hline 11 & 11 & 11.3 & 11.7 & 17.3 & 48 \\
\hline 12 & 12 & 12 & 12 & 17.3 & 49 \\
\hline 13 & 13 & 13 & 13.1 & 18.9 & 49 \\
\hline
\end{tabular}

are demonstrated in Fig. 3 when the multicast group size (counting the source node) $K+1$ varies from 2 (Unicast) to the nodes' number of the network (Broadcast). 5000 multicast sessions are randomly generated for a given multicast group size, meanwhile, Member-Only and Reroute-to-Source algorithms are employed to compute the multicast lightforest for each session. Among 5000 light-forests, the biggest cost of the light-forests (denoted by R2S-Max and MO-Max) and smallest cost of the light-forests (denoted by R2S-Min and MO-Min) are figured out and plotted in Fig. 3. The lower bound and the upper bound provided in Theorem 1]are compared with the simulation result. According to the figure, it is observed that the proposed lower bound is covered by MO-Min since they are almost the same. The lower bound is also very near to R2S-Min. Meanwhile, the upper bound is much higher than the biggest costs obtained (MO-Max and R2S-Max) by the simulation. This can be explained by the fact that the simulation results depend on the simulation topology. The proposed upper bound is valid for all the algorithms which complies the three rules mentioned in Section III As discussed in Subsection [II-B given the network topology in Fig. 4, both the lower bound and the upper bound can be reached. Moreover, from the numerical results of Fig. 3(a) (b) in two different topologies, we can also see that the maximal nodal degree does not influence the quality of cost bounds.
TABLE III

COMPARISON OF APPROXIMATION RATIOS IN NSF NETWORK

\begin{tabular}{|c||c|c||c|c|}
\hline$|D|=K$ & $\rho^{\prime}(\mathrm{MO})$ & $\rho(\mathrm{MO})$ & $\rho^{\prime}$ (R2S) & $\rho$ (R2S) \\
\hline 2 & 2.50 & 1.00 & 2 & 1.13 \\
\hline 3 & 4.5 & 1.03 & 3 & 1.16 \\
\hline 4 & 7 & 1.00 & 4 & 1.18 \\
\hline 5 & 9 & 1.03 & 5 & 1.23 \\
\hline 6 & 8 & 1.04 & 6 & 1.11 \\
\hline 7 & 7 & 1.03 & 7 & 1.32 \\
\hline 8 & 6.13 & 1.07 & 6.13 & 1.35 \\
\hline 9 & 5.44 & 1.06 & 5.44 & 1.29 \\
\hline 10 & 4.9 & 1.03 & 4.9 & 1.39 \\
\hline 11 & 4.45 & 1.04 & 4.45 & 1.54 \\
\hline 12 & 4.08 & 1.00 & 4.08 & 1.45 \\
\hline 13 & 3.77 & 1.01 & 3.77 & 1.46 \\
\hline
\end{tabular}

\section{B. Approximation Ratio of Multicast Light-trees}

In the unweighted NSF network, ILP formulations are carried out to search the optimal light-trees for each multicast session. Provided a multicast group size, 20 random sessions are generated. Hence, each cost is the average of 20 sessions with the same group size. The cost bounds (LB and $\mathbf{U B}$ ) and the approximation ratios of the Rerouteto-Source and Member-Only algorithms are compared in Tables $\Pi$ and III. $\rho^{\prime}(\mathrm{MO})$ denotes the upper bound of the approximation ratio given in Theorem 6 and $\rho^{\prime}$ (R2S) stands for the upper bound of the approximation ratio in Theorem 4 while $\rho(\mathrm{MO})$ and $\rho(\mathrm{R} 2 \mathrm{~S})$ indicate the approximation ratios obtained by $c(M O) / c(I L P)$ and $c(R 2 S) / c(I L P)$ respectively in the simulations. As shown in Table II, Member-Only algorithm has a cost close to the result of the ILP solution. In Table III it is observed that Member-Only algorithm has a better approximation ratio than Reroute-to-Source al gorithm in the simulation. However, the approximation ratio gotten from the simulations is much smaller than that derived from the proof. This result can be explained as follows. First, the approximation ratio derived from the proof is the ratio of the worst case. Second, similar to the cost bound, the approximation ratio depends also on the network topology. Finally, the approximation ratios given in Theorems 4 and 6 are not tight enough.

In fact, another important impact is the characteristic of unweighted NSF network, which plays an important role in 
TABLE IV

NEW Approximation RATIOS OF R2S AND MO IN NSF NETWORK

\begin{tabular}{|c||c|c|c|c|c|c|c|c|c|c|c|}
\hline$|D|=K$ & 2 & 3 & 4 & 5 & 6 & 8 & 9 & 10 & 11 & 12 & 13 \\
\hline $\bar{\rho}(\mathrm{MO})$ & 2.5 & 3 & 3 & 3 & 3 & 3 & 3 & 3 & 3 & 3 & 3 \\
\hline $\bar{\rho}(\mathrm{R} 2 \mathrm{~S})$ & 2 & 3 & 3 & 3 & 3 & 3 & 3 & 3 & 3 & 3 & 3 \\
\hline
\end{tabular}

hel ping Member-Only and Reroute-to-Source to get good performances. This can be explained by the following Lemma 4 .

Lemma 4: Given that WDM network $G$ is unweighted, the approximation ratios of Member-Only and Reroute-to-Source are inferior to the diameter of network $\operatorname{Diam}(G)$.

Proof: Any shortest path $S P_{G}(\cdot)$ in the network $G$ is always $S P_{G}(\cdot) \leq \operatorname{Diam}(G)$. Both Reroute-to-Source and Member-Only algorithm exclusively make use of the shortest path in the network. Thus, the total cost $c(L F)$ of the resultant light-forest is

$$
c(L F) \leq K \times \operatorname{Diam}(G)
$$

Besides, there are $K$ destinations in session $m s(s, D)$ and $G$ is unweighted, the optimal cost of multicast light-trees is always no less than $K$. Thus,

$$
\rho(L F) \leq K \times \operatorname{Diam}(G) / K=\operatorname{Diam}(G)
$$

The diameter of the unweighted NSF network is $\operatorname{Diam}(N S F)=3$. By taking Theorems 3, 5] and Lemma 4 into consideration concurrently, pretty better approximation ratios $\bar{\rho}(M O)$ and $\bar{\rho}(R 2 S)$ can be found in Table $\mathbb{I V}$.

\section{CONCLUSION}

In this work, the problem of all-optical multicast routing is studied theoretically. On one hand, the bounds of wavel ength channel cost used by a multicast session is investigated in unweighted WDM mesh topologies and rings. On the other hand, the performance of two famous AOMR heuristic al gorithms, namely Reroute-to-Source and Member-Only [9], are mathematical evaluated by deriving the approximation ratio, which is a crucial factor of heuristic algorithms. Numerical results in candidate WDM backbone networks demonstrate that the cost bounds and approximation ratios of the Member-Only and the Reroute-to-Source algorithms are far away from those of the worst cases. In addition, the light-forest computed by the Member-Only algorithm uses less cost than that of the Reroute-to-Source algorithm.

In practice, MI nodes and MC nodes have different costs, which is ignored in this paper. One possible future direction can be to develop efficient AOMR heuristic algorithms while take into account the cost associated with the usage of different OXCs. Moreover, incorporating the maximum degree of $M C$ nodes in the network may help to get more precise approximation ratios.

\section{APPENDIXA}

\section{A. Proof of Lemma 1}

Proof: According to Eq. (4), all the $k$ subsets of destinations $D_{i}, i \in[1, k]$, are disjoint. Based on the third assumption in Subsection $11-\mathrm{A}$ at least $k-1$ destinations are not included in a light-tree. The number of nodes in a lighttree is consequently no more than $N-(k-1)$. Furthermore,

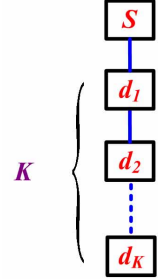

(a)

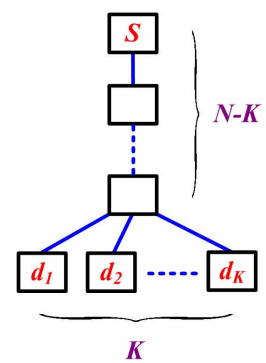

(b)

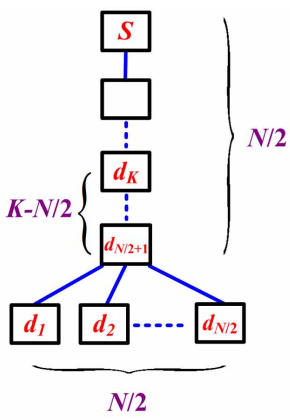

(c)
Fig. 4. (a) The best case; (b) The worst case when $K<\frac{N}{2}$; (c) The worst case when $K \geq \frac{N}{2}$. All the branching nodes are MI nodes.

if no other nodes are included in the $j^{\text {th }}$ light-tree except the source $s$ and the destinations in $D_{j}$ (i.e. $\left\{v \mid v \in L T_{j}\left(s, D_{j}\right)\right\}$ $\left.=\{s\} \cup D_{j}\right)$, then the number of nodes in the $j^{t h}$ light-tree is minimal and equals $K_{j}+1$. Hence, the cost bounds of a light-tree can be obtained as

$$
K_{j} \leq c\left(L T_{j}\left(s, D_{j}\right)\right) \leq N-k
$$

\section{B. Proof of Theorem 1}

Proof: According to Lemma 1 and Eq. (6), the total cost of the light-trees built for a multicast session $m s(s, D)$ holds

$$
\begin{aligned}
\mathcal{C}(m s(s, D)) & \leq \sum_{i=1}^{k}(N-k) \\
& \leq k(N-k) \\
& \leq-\left(k-\frac{N}{2}\right)^{2}+\frac{N^{2}}{4}
\end{aligned}
$$

Regarding $k$ is an integer and $1 \leq k \leq K$, we obtain

$$
\mathcal{C}(m s(s, D)) \leq \begin{cases}K(N-K), & K<\frac{N}{2} \\ \frac{N^{2}}{4}, & K \geq \frac{N}{2} \text { and } N \text { is even } \\ \frac{N^{2}-1}{4}, & K \geq \frac{N}{2} \text { and } N \text { is odd }\end{cases}
$$

Moreover, according to Lemma 1, it is also true that

$$
\mathcal{C}(m s(s, D)) \geq \sum_{i=1}^{k} K_{i}=K
$$

In the following, we prove that the cost bounds in Theorem 1 are tight. It is not difficult to imagine that the case with the minimal cost appears when all and only all the destinations are involved in the light-tree computed for multicast session $m s(s, D)$, as shown in Fig. 4(a). That is to say $\{v \mid v \in L T\}=\{s\} \cup D$. It is obvious that the lower bound $K$ is tight. The worst case depends on the relationship between $K$ and $N$. In case that $K<\frac{N}{2}$, the worst case may happen when the network topology is like that in Fig. 4(b), where $K$ lightpaths on different wavelengths are needed to serve $K$ destinations to the source. Here, it is observed that the cost of the optimal light-trees equals $K(N-K)$. When $K \geq \frac{N}{2}$, the worst case may take place in the topology of Fig. 4 (c). In this topology, $\left\lfloor\frac{N}{2}\right\rfloor$ lightpaths from the source to each of the destinations at the bottom are required to serve all the group members. The $K-\left\lfloor\frac{N}{2}\right\rfloor$ destinations in the middle can be served in any one of them. As each lightpath 


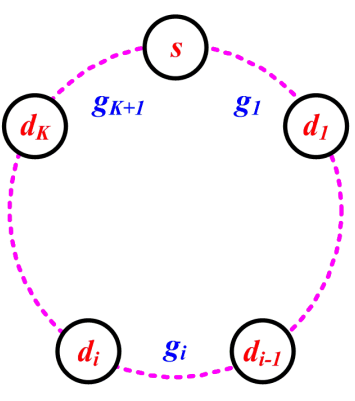

Fig. 5. The gaps in a WDM ring

has a cost of $\left\lceil\frac{N}{2}\right\rceil$, an exact total cost of $\left\lfloor\frac{N^{2}}{4}\right\rfloor$ should be consumed to establish the multicast session $m s(s, D)$. This example verifies the accuracy of the upper bound given in Theorem 1 ]

\section{Proof of Theorem 2}

Proof: Beginning from the source node $s$, we index the destination nodes from $d_{1}$ to $d_{K}$ in a clockwise manner. Let $g_{1}$ denote the length of the gap between the source $s$ and $d_{1}$, $g_{i}$ be the length of the $i^{\text {th }}$ gap, i.e., the gap between $d_{i-1}$ and $d_{i}$, and $g_{K+1}$ be the gap between source $s$ and $d_{K}$ as shown in Fig. [5] In a WDM ring of $N$ nodes, we obtain

$$
\sum_{i=1}^{K+1} g_{i}=N
$$

The cost of the optimal multicast light-tree for multicast session $m s(s, D)$ can be determined by

$$
\mathcal{C}(m s(s, D))=N-\max _{1 \leq i \leq K+1} g_{i}
$$

In order to obtain the cost bound of the light-tree, we have to determine the value range of $\max _{1 \leq i \leq K+1} g_{i}$. Note that all $g_{i}$ are positive integers and satisfy Eq. (42). We obtain the following inequality

$$
\max _{1 \leq i \leq K+1} g_{i} \geq\left\lceil\frac{N}{K+1}\right\rceil
$$

This result corresponds to the case that multicast members are evenly distributed in a WDM ring. Thus we obtain

$$
\mathcal{C}(m s(s, D)) \leq N-\left\lceil\frac{N}{K+1}\right\rceil
$$

Besides, if all the multicast group members stick together one by one, the optimal light-tree thus only consists of the source and the destinations. Then, we can obtain the lower bound

$$
\mathcal{C}(m s(s, D)) \geq K
$$

\section{APPENDIX B}

\section{A. Proof of Theorem 3}

Proof: Let $r_{\max }$ be the cost of the shortest path from the furthest destination to the source $s$, i.e.

$$
r_{\text {max }}=\max _{d_{i} \in D} c\left[S P\left(s, d_{i}\right)\right]
$$

Obviously, we have

$$
C_{O p t} \geq r_{\max }
$$

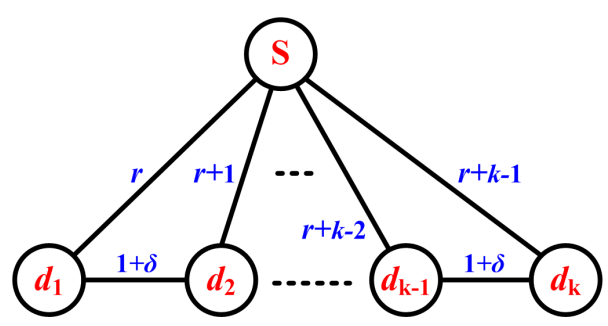

Fig. 6. Illustration of Theorem 3

Hence, we can obtain

$$
\begin{aligned}
\rho(R 2 S) & =\mathcal{C}(R 2 S) / C_{O p t} \\
& \leq \sum_{d_{i} \in D} c\left(S P\left(s, d_{i}\right)\right) / C_{O p t} \\
& \leq|D| \cdot r_{\max } / r_{\max } \\
& \leq K
\end{aligned}
$$

Next, we will show that $\rho(R 2 S)$ may tend to be $K$ in a nonequally-weighted topology like Fig. 6, where $r$ is a positive integer denoting the distance from $s$ to $d_{1}$ and $\delta$ is a very small non-negative number. We can see the optimal solution for multicast communication $m s\left(s, d_{1}-d_{K}\right)$ is the lightpath $s \rightarrow d_{1} \rightarrow d_{2} \ldots \rightarrow d_{K}$, while the shortest path tree is the set of direct paths from $s$ to each destination. Then,

$$
\begin{gathered}
\mathcal{C}(R 2 S)=K\left(r+\frac{K-1}{2}\right) \\
C_{O p t}=r+(K-1)(1+\delta)
\end{gathered}
$$

Thus, the approximation ratio of $\mathrm{R} 2 \mathrm{~S}$ algorithm is

$$
\rho(R 2 S)=K\left(1-\frac{1}{\frac{2 r}{(K-1)(1+2 \delta)}+\frac{2(1+\delta)}{1+2 \delta}}\right)
$$

Since $G$ is non-equally weighted and $K$ is inferior to $N, r$ can be arbitrarily large and independent of $K$ and $N$. Thus, for any $K \in(1, N)$, when $\frac{r}{N} \rightarrow \infty$ and $\delta \rightarrow 0$, we obtain $\rho(R 2 S)=K$.

\section{Discussion:}

Obviously, Theorem 3 , i.e. $\rho(R 2 S) \leq K$ is true for both unweighted and non-equally-weighted networks $G$. However, it should be noticed that $\rho(R 2 S)=K$ is not valid for all possible $1<K<N$ in unweighted WDM networks, especially when $K$ is very dose to $N$. Take the same example in Fig. 6] if $G$ is unweighted, $r$ is always below $N-K$ and $\delta=0$, thus $\frac{r}{K} \leq \frac{N-K}{K}$ will never reach $\infty$ when $K$ is close to $N$. As a result, Eq. (52) can not tend to $K$ any more, and a better ratio should be found in this case.

\section{B. Proof of Theorem 4}

Proof: As proved in Theorem [3] that $\rho(R 2 S) \leq K$ is always true for any WDM networks. Combining this with Lemma 2, the proof follows.

\section{Lemma 5}

Lemma 5: In Fig. 7 suppose $P$ is a node in the shortest path $S P(A, B)$ from node $A$ to node $B$, and $C$ is connected to $P$ by the shortest path. Define $l_{X Y}$ as the cost of the shortest path $S P(X, Y)$, and we obtain

$$
l_{C P} \leq \frac{1}{2}\left(l_{A B}+l_{A C}+l_{B C}\right)
$$




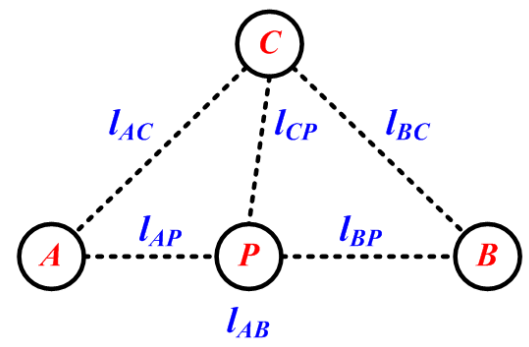

Fig. 7. Illustration of Lemma 5

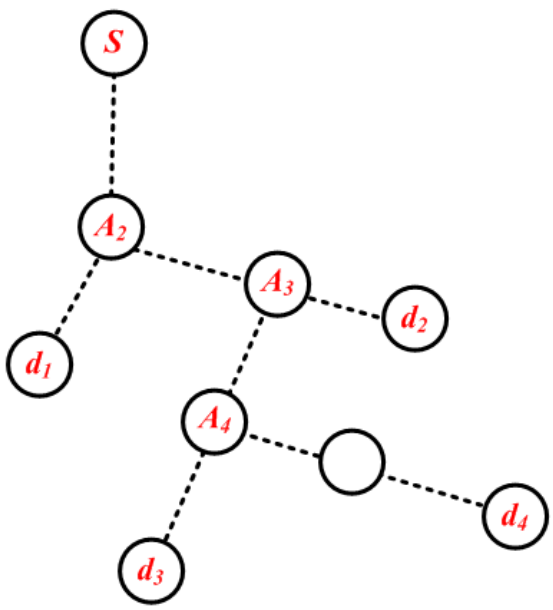

Fig. 8. Demonstration of the worst case of the Member-Only algorithm

Proof: Since node $P$ is in $S P(A, B)$, both paths $A P$ and $B P$ are the shortest paths, then

$$
l_{A B}=l_{A P}+l_{B P}
$$

As a result the graph in Fig. 7 is a distance network, where the triangle inequality is valid. Then,

$$
\begin{aligned}
& l_{C P} \leq l_{A C}+l_{A P} \\
& l_{C P} \leq l_{B C}+l_{B P}
\end{aligned}
$$

Adding Eq. [55] to Eq. (56) gives

$$
2 l_{C P} \leq\left(l_{A P}+l_{B P}\right)+l_{A C}+l_{B C}
$$

By substituting Eq. (54) into the above equation, Lemma 5 follows.

\section{Proof of Theorem 5}

Proof: We use the proof by induction. Let $l_{\max }$ be the cost of the shortest path between the furthest two members in a multicast session $m s(s, D)$, i.e.

$$
l_{\max }=\max _{m_{i}, m_{j} \in s \cup D} c\left[S P\left(m_{i}, m_{j}\right)\right]
$$

Member-Only algorithm starts the multicast light-tree $L T$ from the source $s$ and spans the light-trees iteratively. Let $l_{i}$ denote the cost of the shortest path that connects the destination $d_{i}$ to the current $L T$, and $l_{i}^{m}$ be its upper bound. In other words, the cost of $L T$ increases by $l_{i}$ after spanning $d_{i}$, and at most $l_{i}^{m}$. In the following, we are trying to determine the worst case of the upper bound $l_{i}^{m}$ for each $l_{i}$ by applying the triangle inequality in Lemma [5. As shown in Fig. [8, the nearest destination node $d_{1}$ to the source $s$ is first added to $L T$. Now, the cost of $L T$ is $l_{1} \leq l_{\max }$ and $l_{1}^{m}=l_{\max }$. Then in the second step, the nearest destination $d_{2}$ to $L T$ is added using the shortest path. If $d_{2}$ is spanned via $d_{1}$ or $s$, then obviously $l_{2} \leq l_{\max }$. It should be noted that the worst case appears when $d_{2}$ is spanned via an intermediate node (say $A_{2}$ ) in $S P\left(s, d_{1}\right)$. If this happens to be the case, we obtain $l_{2} \leq \frac{3}{2} l_{\max }$ and $l_{2}^{m}=\frac{3}{2} l_{\max }$ according to Lemma 5, In the third step, the nearest destination $d_{3}$ is added using the shortest path. It is evident that $l_{3}^{m}$ is the largest when $d_{3}$ is spanned via an intermediate node (say $\left.A_{3}\right)$ in $S P\left(A_{2}, d_{2}\right)$. This can be explained as follows. If $d_{3}$ is spanned via any member node (i.e., $s, d_{1}$ or $d_{2}$ ), then obviously $l_{3} \leq l_{\max }$. Otherwise, $d_{3}$ must be connected via an intermediate node in the shortest path $S P\left(s, d_{1}\right)$ or $S P\left(A_{2}, d_{2}\right)$. According to Lemma [5] $l_{3} \leq \frac{3}{2} l_{\max }$ if $d_{3}$ connects to $L T$ through a node in $S P\left(s, d_{1}\right)$. In case that $d_{3}$ connects to $L T$ through a node in $S P\left(A_{2}, d_{2}\right)$, the cost of $S P\left(A_{2}, d_{3}\right)$ should be calculated before using the triangle inequality. Similar to $\operatorname{SP}\left(A_{2}, d_{2}\right)$, $c\left[S P\left(A_{2}, d_{3}\right)\right] \leq l_{2}^{m}$. Then, go back to $l_{3}$, and we obtain:

$$
\begin{aligned}
l_{3} & \leq \frac{1}{2}\left(c\left[S P\left(A_{2}, d_{3}\right)\right]+c\left(S P\left(d_{2}, d_{3}\right)\right)+l_{2}\right) \\
& \leq \frac{1}{2}\left(l_{2}^{m}+l_{\max }+l_{2}\right) \\
& \leq l_{2}^{m}+\frac{1}{2} l_{\max }
\end{aligned}
$$

Hence,

$$
l_{3}^{m}=l_{2}^{m}+\frac{1}{2} l_{\max }
$$

Suppose that Eq. 61] is obtained by applying Lemma [5

$$
l_{i}^{m}=l_{i-1}^{m}+\frac{1}{2} l_{\max }
$$

Next, we try to prove that it is also true for the case of $l_{i+1}^{m}$. Since a Member-Only multicast light-tree is only consisted of the shortest paths, each node in the light-tree must be in the shortest path between two member nodes or between a destination and a joint node of two shortest paths. And, $l_{i}^{m}$ is monotonically increasing. Consequently, the worst case of $l_{i+1}^{m}$ occurs when $d_{i+1}$ connects to $L T$ through an intermediate node in the shortest path between $d_{i}$ and a joint node $A_{i}$. According to Lemma [5] $c\left[S P\left(A_{i}, d_{i+1}\right)\right] \leq l_{i}^{m}$ also holds. Then, applying the triangle inequality again in the distance network of $G\left(A_{i}, d_{i}, d_{j}\right)$ leads to,

$$
\begin{aligned}
l_{i+1} & \leq \frac{1}{2}\left(c\left[S P\left(A_{i}, d_{i+1}\right)\right]+c\left(S P\left(d_{i}, d_{i+1}\right)\right)+l_{i}\right) \\
& =\frac{1}{2}\left(l_{i}^{m}+l_{\max }+l_{i}\right) \\
& \leq l_{i}^{m}+\frac{1}{2} l_{\max }
\end{aligned}
$$

So, it is always valid for all the steps during the span of a light-tree that $l_{i+1}^{m}=l_{i}^{m}+\frac{1}{2} l_{\max }$. Hence, we have $l_{i}^{m}=\frac{i+1}{2} l_{\max }$. Assuming $k$ light-trees are constructed for multicast session $m s(s, D)$, and $\left|D_{i}\right|$ destinations are unique served in the $i^{\text {th }}$ light-tree. This also means that $\left|D_{i}\right|$ steps are processed in the $i^{\text {th }}$ light-tree. Thus, the total cost of the 
$i^{\text {th }}$ light-tree is upper bounded by

$$
\begin{aligned}
c\left(L T_{i}\right) & =\sum_{i=1}^{\left|D_{i}\right|} l_{i} \\
& \leq \sum_{i=1}^{\left|D_{i}\right|} l_{i}^{m} \\
& \leq \frac{1}{4}\left(\left|D_{i}\right|^{2}+3\left|D_{i}\right|\right) l_{\max }
\end{aligned}
$$

Then, the total cost consumed by $m s(s, D)$ using MemberOnly algorithm is

$$
\begin{aligned}
\mathcal{C}(M O) & =\sum_{i=1}^{k} c\left(L T_{i}\right) \\
& \leq \sum_{i=1}^{k} \frac{1}{4}\left(\left|D_{i}\right|^{2}+3\left|D_{i}\right|\right) l_{\max } \\
& \leq \frac{1}{4}\left(3|D|+\sum_{i=1}^{k}\left|D_{i}\right|^{2}\right) l_{\text {max }} \\
& \leq \frac{1}{4}\left(3|D|+|D|^{2}\right) l_{\text {max }}
\end{aligned}
$$

As $C_{O p t} \geq l_{\max }$, the following inequality can be obtained

$$
\begin{aligned}
\rho(M O) & =\mathcal{C}(M O) / C_{O p t} \\
& \leq \mathcal{C}(M O) / l_{\max } \\
& \leq \frac{1}{4}\left(3 K+K^{2}\right)
\end{aligned}
$$

\section{E. Proof of Theorem 6}

Proof: By merging the approximation ratios in Lemma 2 and Theorem [5, the proof follows.

\section{REFERENCES}

[1] Ashraf Hamad, Tao Wu, Ahmed E. Kamal, Arun K. Somani. On multicasting in wavelength-routing mesh networks. Computer Networks, vol. 50, no. 15, pp. 3105-3164, 2006

[2] Laxman H. Sahasrabuddhe, Biswanath Mukherjee. Light-trees: optical multicasting for improved performance in wavelengthrouted networks. IEEE Communications Magazine, vol. 37, no. 2, pp. 67-73, 1999.

[3] R. Malli, Xijun Zhang, Chunming Qiao. Benefit of multicasting in all-optical networks. SPIE Proceeding on All-Optical Networking, 1998, pp. 209-220.

[4] Maher Ali, J itender S. Deogun. Cost-effective implementation of multicasting in wavelength-routed networks. IEEE/OSA J ournal of Lightwave Technology, vol. 18, no. 12, pp. 1628-1638, 2000.

[5] Fen Zhou, Miklós Molnár, Bernard Cousin, Chunming Qiao. Cost bounds of multicast light-trees in WDM networks. The $9^{t h}$ IFIP International Conference on Networking (Networking10), Chennai, India, 2010, pp. 94-105.

[6] Fen Zhou, Miklós Molnár, Bernard Cousin, Chunming Qiao. Approximation ratios of multicast light-trees in WDM networks. IEEE Global Communications Conference (GLOBECOM 10), Miami, USA, 2010, pp. 1-6.

[7] Xiaohua Jia, Dingzhu Du, Xiaodong Hu, Mankei Lee, J un Gu. Optimization of wavelength assignment for QOS multicast in WDM networks. IEEE Transaction on Communications, vol. 49, no. 2, pp. 341-350, 2001.

[8] Biswanath Mukherjee. WDM optical communication networks: progress and challenges. IEEE J ournal on Selected Areas in Communications, vol. 18, no. 10, pp. 1810-1824, 2000.

[9] Xijun Zhang, J ohn Wei, Chunming Qiao. Constrained multicast routing in WDM networks with sparse splitting. IEEE/OSA J ournal of Lightware Technology, vol. 18, no. 12, pp. 1917-1927, 2000 .
[10] Fen Zhou, Miklós Molnár, Bernard Cousin. Avoidance of multicast incapable branching nodes in WDM netwoks. Photonic Network Communications, vol. 18, no. 3, pp. 378-392, 2009.

[11] Fen Zhou, Miklós Molnár, Bernard Cousin. Is light-tree structure optimal for multicast routing in sparse light splitting WDM networks? The $18^{\text {th }}$ International Conference on Computer Communications and Networks (IC3N '09), San Francisco, USA, 2009, pp. 1-7.

[12] Cheng-Yu Hsieh and Wanjiun Liao. All-optical multicast routing in sparse splitting WDM networks. IEEE J ournal On Se lected Areas in Communications, vol. 25, no. 6, pp. 51-62, 2007.

[13] Hwachun Lin, Shengwei Wang. Splitter placement in alloptical WDM networks. IEEE Global Communications Conference (GLOBECOM 05), St. Louis, USA, 2005, pp. 306-310.

[14] H. Takahashi, A. Matsuyama. An approximate solution for the Steiner problem in graphs. Math. J aponica, vol. 24, no. 6, pp. 573-577, 1980.

[15] Pawel Winter. Steiner problem in networks: a survey. Networks, vol. 17, no. 2, pp. 129-167, 1987.

[16] L. Kou, G. Markowsky, L. Berman. A fast algorithm for Steiner trees. Acta Informatica, vol. 15, no. 2, pp. 141-145, 1981.

[17] Michael Scheutzow, Patrick Seeling, Martin Maier, and Martin Reisslein. Multicasting in a WDM-upgraded resilient packet ring. J ournal of Optical Networking, vol. 6, no. 5, pp. 415-421, 2006.

[18] Michael Scheutzow, Patrick Seeling, Martin Maier, and Martin Reisslein. Shortest path routing in optical WDM ring networks under multicast traffic. IEEE Communications Letters, vol. 10, no. 7, pp. 564-566, 2006.

[19] Oliver Yu, Yuan Cao. Mathematical formulation of optical multicast with loss-balanced light-forest. IEEE Global Communications Conference (GLOBE COM 05), St. Louis, USA, 2005, pp. 1788-1792.

[20] Ming-Tsung Chen, B.M.T. Lin, Shian-Shyong Tseng. Multicast routing and wavelength assignment with delay constraints in WDM networks with heterogeneous capabilities. J ournal of Network and Computer Applications, vol. 31, no. 1, pp. 47-65, 2008.

[21] http://www.ilog.com/products/cplex/.

[22] http://www.algorithmic-solutions.com/leda/index.htm 